\title{
Optimization of Enterprise Labor Resource Allocation Based on Quality Optimization Model
}

\author{
Gaoyang Liang, ${ }^{1}$ Long $X u\left(\mathbb{D},{ }^{2}\right.$ and Liang Chen ${ }^{2}$ \\ ${ }^{1}$ School of Labor Economics, Capital University of Economics and Business, Beijing 100070, China \\ ${ }^{2}$ School of Business Administration, Hebei University of Economics and Business, Shijiazhuang, Hebei 050061, China \\ Correspondence should be addressed to Long Xu; xulong@heuet.edu.cn
}

Received 5 February 2021; Revised 27 February 2021; Accepted 30 March 2021; Published 10 April 2021

Academic Editor: Wei Wang

Copyright $(92021$ Gaoyang Liang et al. This is an open access article distributed under the Creative Commons Attribution License, which permits unrestricted use, distribution, and reproduction in any medium, provided the original work is properly cited.

Companies take project-based management as their organizational strategy, and project quality assurance plays a vital role in improving customer satisfaction and enhancing corporate image. Starting from the perspective of optimizing project quality, this paper assigns different quality influencing factors to each project and each task of the project, divides the labor resources shared by multiple projects in the enterprise according to the skill level, and transforms the problem of project quality optimization. The problem of the highest skill level of labor resources allocated to all projects of the enterprise is designed, and algorithms are designed to achieve the optimization of project quality through the optimal allocation of labor resources. The various links in this article are closely related to form a comprehensive, scientific, and systematic research system for optimal human resource allocation, human resource management, and development. Finally, case analysis is used to confirm the usability of the model and provide a quantitative method and perspective for project-oriented companies to allocate workforce.

\section{Introduction}

Every step of human survival and development is inseparable from the support of resources. An enterprise is a product that accompanies human development. At the same time, it is the creator of human material wealth. All production and operation activities of an enterprise cannot be separated from natural resources and capital resources, labor resources, and technical resources [1]. An enterprise's position and competitiveness in market competition often depend on the degree of possession of these three resources, namely, the quantity, quality, and structure of resources. Throughout the world, the development of many enterprises has almost one thing in common; that is, "labor resources" are regarded as the core resource of the enterprise and determined as the support point of social, economic, and technological development, and this labor resource has been used by the society [2]. In today's society, labor resources have become a science and become the first resource for scientific and technological progress and enterprise development. Small- and medium-sized enterprises, a group that accounts for more than $98 \%$ of the number of Chinese enterprises, have ushered in more opportunities and challenges, but the ensuing financial crisis has also forced the group of small- and medium-sized enterprises to face more severe survival and development challenges.

The competition in the 21st century is mainly the competition of talents. If small- and medium-sized enterprises want to survive and develop in the fierce competition, it depends to a large extent on the company's labor resource allocation [3]. With the turbulence of the world economic environment, in recent years, Chinese enterprises have gradually begun to face the issue of labor resources. The allocation of labor resources of enterprises has also become one of the hot topics of concern to experts, entrepreneurs, and society in labor resources [4]. What method should small- and medium-sized enterprises choose to enhance their core competitiveness in order to maintain their advantages and develop healthily in a fiercely competitive environment has become the answer that business managers desire. In theory and practice, with the continuous improvement of labor resource management, the efficiency of 
labor resource allocation has become the focus of attention and an important dimension to measure the efficiency of labor resource management [5].

The specific content is as follows: first, we explain the research background, purpose, and significance of this article. With the intensification of market competition, the market space and survival pressure of small- and mediumsized enterprises continue to rise, and the allocation of labor resources has gradually been paid attention to by experts, scholars, and business managers and has become an important dimension to measure the effectiveness of labor resource management. Then, it expounds the current research status of labor resource management at home and abroad, combs the related theories of labor resource allocation at home and abroad, and shows the theories based on this research: the theory of person-post matching and the theory of person-organization matching. Then, starting with the definition and division of small and medium-sized enterprises, combined with the characteristics of small- and medium-sized enterprises, it analyzes the common problems that appear in the labor resource management of most of the small- and medium-sized enterprises in our country. The research idea in this article is to combine characteristics and development to analyze the importance of labor resource allocation efficiency in the development of SME (small- and medium-sized enterprises) through the development of relevant theories and literature on labor resource allocation at home and abroad. Use companies as research targets for trends and analysis. The current state of labor resource management shows several ways to improve the efficiency of labor resource allocation in enterprises.

\section{Related Work}

Among the related researches on the allocation of labor resources in state-owned enterprises in my country, representative studies include the following. Wang and Yang [6] use key variables into the tree modeling method, through linear causality analysis, to create the overall labor resources in state-owned enterprises in our country incentive and feedback the causal structure model, and then analyze the relationship and the mechanism of action between employee satisfaction and performance appraisal mechanism through the analysis of the control archetype. The research mainly focuses on the microfields of labor resource allocation in our state-owned enterprises and does not systematically study labor resource allocation as a whole from the macro perspective. Zhang and others [7] proposed to improve the allocation of labor resources and incentive mechanisms on the basis of the bank's property rights system and internal governance structure. The research mainly focuses on qualitative analysis and research. The present situation and goals of labor resource allocation are all based on qualitative analysis. Professor Zhao et al. [8] focus on the qualitative analysis that restricts the demand and supply of labor resources in state-owned enterprises in our country. They comprehensively analyze the factors that affect the supply and demand of labor resources in state-owned enterprises in our country from both economic and noneconomic factors.
They also analyze the balance between the supply and demand of labor resources in state-owned enterprises.

In today's fierce competition, the importance of labor resources is self-evident compared to all kinds of resources in enterprises. Simply put, the allocation of labor resources is how to make full use of the company's own labor resources and allocate them as reasonably and effectively as possible [9]. As an important aspect of labor resources management, it will play a bridge role in linking the development and utilization of labor resources.

The so-called enterprise labor resource allocation refers to the timely and reasonable arrangement of all kinds of talents that meet the needs of enterprise development through assessment, selection, recruitment, and training in the required positions, so that they can be combined with other economic resources to form a realistic economic movement that allows people to make the best use of their talents, increase the productivity of labor resources, and maximize the creation of more economic and social benefits for enterprises and society [10].

Most companies simply consider whether their existing workforce matches the number required for production and operation that is the subject of a company's workforce allocation survey. Today, a common practice for most small businesses is to "hire an employee after quitting a job" with little consideration of the quality and structure of the allocation. However, these two aspects are the essence and core of efficient workforce allocation. A deeper level of workforce allocation is related to the quality and resource structure of a company's workforce. Job assignment specifically refers to whether the existing workforce education and training status are consistent with the workforce and whether the company's workforce council is rational and efficient. The so-called labor resource allocation efficiency refers to finding the most matching combination of labor resource elements in continuous attempts and investing it in the production of enterprises, and the purpose is to hope to get the greatest benefit. However, in actual production, the "best" is almost impossible to achieve, so as far as the enterprise is concerned, it is only necessary to make full use of the existing labor resources under the condition of constant input to improve efficiency and input-output ratio [11].

\section{Principles of Enterprise Labor Resource Allocation}

Due to the scarcity and value of resources, effective allocation of human resources to avoid resource conflicts between projects and improve resource utilization efficiency plays a vital role in the successful implementation of projects. Project-oriented enterprises must fully tap the potential of the company's labor resources, make the best use of their talents, ensure the supply of project labor resources, promote the effective allocation of resources among project activities, reduce labor resource costs, and maximize full play to the role of labor resources [12]. However, how to achieve a scientific and reasonable configuration is a question that has been under discussion for a long time. The allocation of labor 
resources for project-oriented enterprises should follow the following principles.

3.1. Overall Strengthening. The optimal allocation of labor resources for project-oriented enterprises should strengthen the overall labor resource functions of the enterprise. From an overall perspective, the labor resources of the entire enterprise can be dynamically and effectively allocated among all the projects of the enterprise, and the labor resource cost of the entire enterprise should be reduced. Improve the project quality of the entire enterprise and maximize the role of human resources.

3.2. Dynamic Adjustment. Project-oriented enterprises are guided by a large number of project activities, which indicates that their labor resources are in a dynamic organizational environment, and personnel need to constantly adjust and optimize allocation among different projects. The dynamic principle means that when personnel or job requirements change, the staffing should be adjusted in a timely manner to ensure that the right people are always working in the right positions.

3.3. Energy Level Correspondence. A person may have multiple skills, each person has a different skill level for different tasks, and each task of each project requires different skill levels for the personnel. The optimal allocation of labor resources should correspond to the energy level. The skill level of each person corresponds to the skill level required by the project and the company's various positions. Person-post matching, in layman's terms, is to select people with qualifications for the post according to the quality requirements of different posts. Experts and scholars define it as based on the six major factors of corporate strategy, corporate scale, corporate development, employee quality, technical factors, and environmental factors, through job analysis and design, description, training, planning, evaluation, incentives and constraints, process control, etc., to achieve the matching between individuals and positions [13]. Specifically, it involves two meanings: one, the required ability requirements of the post need someone to have the complete requirements; two, the ability of a person fully meets the requirements of the post. Its core is to make the matching of people and posts reach the most reasonable state as much as possible and show the optimization. Refined to the enterprise, person-post matching is to select suitable personnel for a given position to match and take effective measures to achieve an effective combination of employees and positions.

Person-post matching is a dynamic change. From the initial general matching state to a high-matching state, and then degenerating from a high-matching state to an initial matching state, this is because, with the needs of development, the quality requirements for the position continue to increase, but the practitioners are standing still. It can only degenerate into the primary level of matching in the end. The flow chart of dynamic person-post matching is shown in Figure 1.

3.4. Comprehensive Plan. When formulating a labor resource allocation plan, the constraints between the resource allocation plan and the choice of project implementation plan, project schedule, cost, and financial plan should be fully considered. The allocation of labor resources must be incorporated into cost management and project schedules, as a dynamic constraint of project cost management and schedule planning. When arranging various project activities, labor resources must be considered. After the project plan is formulated, a detailed resource plan must be made to ensure the implementation of the project plan, or adjust the project plan.

Most scholars broadly and simply define person-organiza tion matching as the compatibility between individuals and organizations. This theoretical study was first proposed by Lewin. After decades of development, the following theories have been formed [14].

(1) Naseri and Navimipour [15] proposed the attractionselection model, and the organizational demography model proposed by Evangelinou et al. [16] had the greatest impact. These two models believe that the employees in the organization are the people who are attracted by the organization and finally retained after the organization's selection. Employees belonging to the same organization are affected by the organization's culture. There will be similarities in the way of performance. Researching and understanding the composition of the organization are the key to understanding the operation of the organization.

(2) Guo and Qui refined the types of matching into two types: consistent matching and complementary matching. The so-called consistency matching means that individuals and organizations have the same or similar characteristics. On the contrary, complementary matching means that both organizations and individuals can meet the needs and requirements of each other [17].

(3) Liu et al. put forward the viewpoint of demandprovide and demand-ability [18]. The theory defines human organization matching as the matching of personal abilities and work needs, that is, when a need is provided, or the matching of personal requirements and work attributes, that is, a requirement for a capability.

(4) The above several viewpoints have studied peopleorganization matching from different aspects, but the considerations are not too comprehensive and relatively single, until $\mathrm{Yu}$ and Solvang proposed a relatively complete human-organization matching model, which combines the above several viewpoints based on theories that define person-organization matching as the compatibility of employees and organizations in three situations [19]. Two 


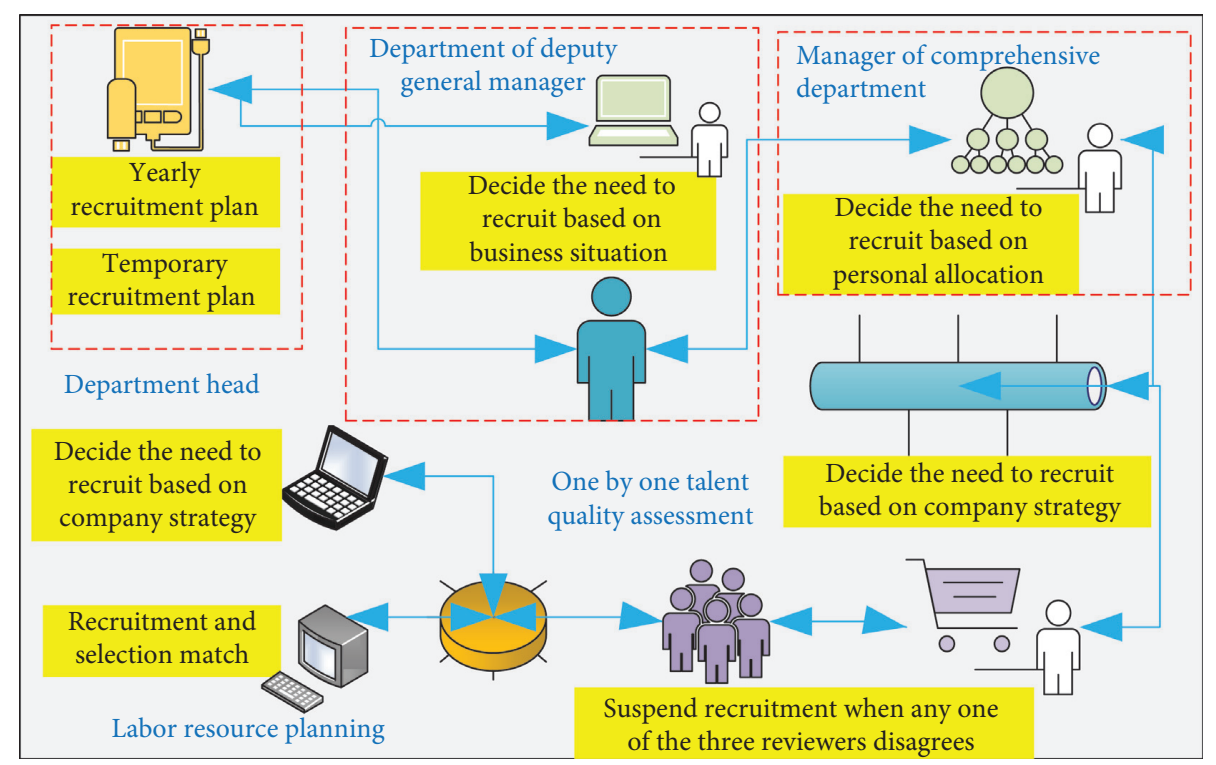

FIgURE 1: Flow chart of dynamic person-post matching.

conditions must be met: one is that at least one of the individuals and organizations as members of the organization can provide for the other the resources it needs; the second is that individuals and organizations have similarities in some basic characteristics. Figure 2 shows conceptual model of person organization matching.

\section{Construction of Labor Resource Allocation Model}

4.1. Problem Description. A project-oriented enterprise has to do $n$ different projects in a certain period of time, forming a project set $Y=\left\{y_{1}, y_{2}, \ldots, y_{n}\right\}$, and the project weight of each project is $W=\left\{w_{1}, w_{2}, \ldots, w_{n}\right\}$. The enterprise gives the project importance evaluation through expert evaluation and fuzzy comprehensive evaluation according to its strategic objectives, project scale, project income, etc. [18]. Project $Y_{i}$ is composed of $N$ different tasks, $Y_{i}=\left\{x_{i 1}, x_{i 2}, \ldots, x_{i j}, \ldots, x_{i n}\right\}, j \in n_{i}, x_{i j}$ represents the $j^{\text {th }}$ task of the $i$-th project; each task requires different work skills; the number of workers and working hours required by each task $x_{i j}$ are $p_{i j}$ and $t_{i j}$ respectively; $t_{i}=\sum_{i=1}^{j} t_{i j}$ represents the duration of the $i$-th project; the quality of each task has an impact factor on the quality of the entire project as $\varphi_{i j}$.

There are $m$ individuals in the enterprise that can be used for the scheduling of these $n$ projects. These $m$ individuals are project team staff and do not belong to the management of the enterprise. The salary level is equivalent [19]. Each person has a variety of skills that cannot be used, but the skill level is different. $\chi_{k l}$ represents the $l$-th skill level possessed by the $k$-th person. The larger the $\chi_{k l}$, the higher the skill level. $\chi_{k l} \in[0,2], 0$ means that the skill is not at all, 1 means that the skill is basically mastered, and 2 means the skill master is quite proficient. The skill level coefficient is evaluated by the labor resources department of the enterprise based on employee data and historical work performance (the expert group fuzzy comprehensive evaluation method can be used). Assuming that these $n$ projects are started at the same time, the projects are completely independent of each other, and there are no prioritization constraints. However, there are prioritization constraints between the various tasks of the project. The subsequent tasks must be completed after the previous tasks are completed. Once a person is assigned a task, they must work until the task is completed, and they cannot be replaced in the middle to ensure that the cost of labor resource scheduling is minimized. In addition, a person can only do one task at a time and cannot hold multiple jobs. Now, it is necessary to assign $m$ individuals to different tasks of $n$ projects under the condition of ensuring uninterrupted project implementation, find a labor resource allocation plan, and clarify the personnel to be assigned each task in each project.

4.2. Optimization Model. In project-oriented enterprises, the enterprise regards project-based management as an organizational strategy, and the quality assurance of projects plays a vital role in improving customer satisfaction and enhancing corporate image. As everyone knows, people, machines, materials, methods, and environment are the five main factors that affect the quality of the project. People play a decisive role in the five factors, and everything is peopleoriented [20]. Therefore, the level of project quality can be indirectly reflected by the ability level of labor resources. The stronger the ability, the higher the quality of the project. Therefore, under the condition that other influencing factors remain unchanged, the enterprise's project quality optimization problem can be transformed into the problem of the greatest skill level of labor resources allocated by all projects of the enterprise. The enterprise's labor resource allocation needs to ensure that a configuration plan is found under the 


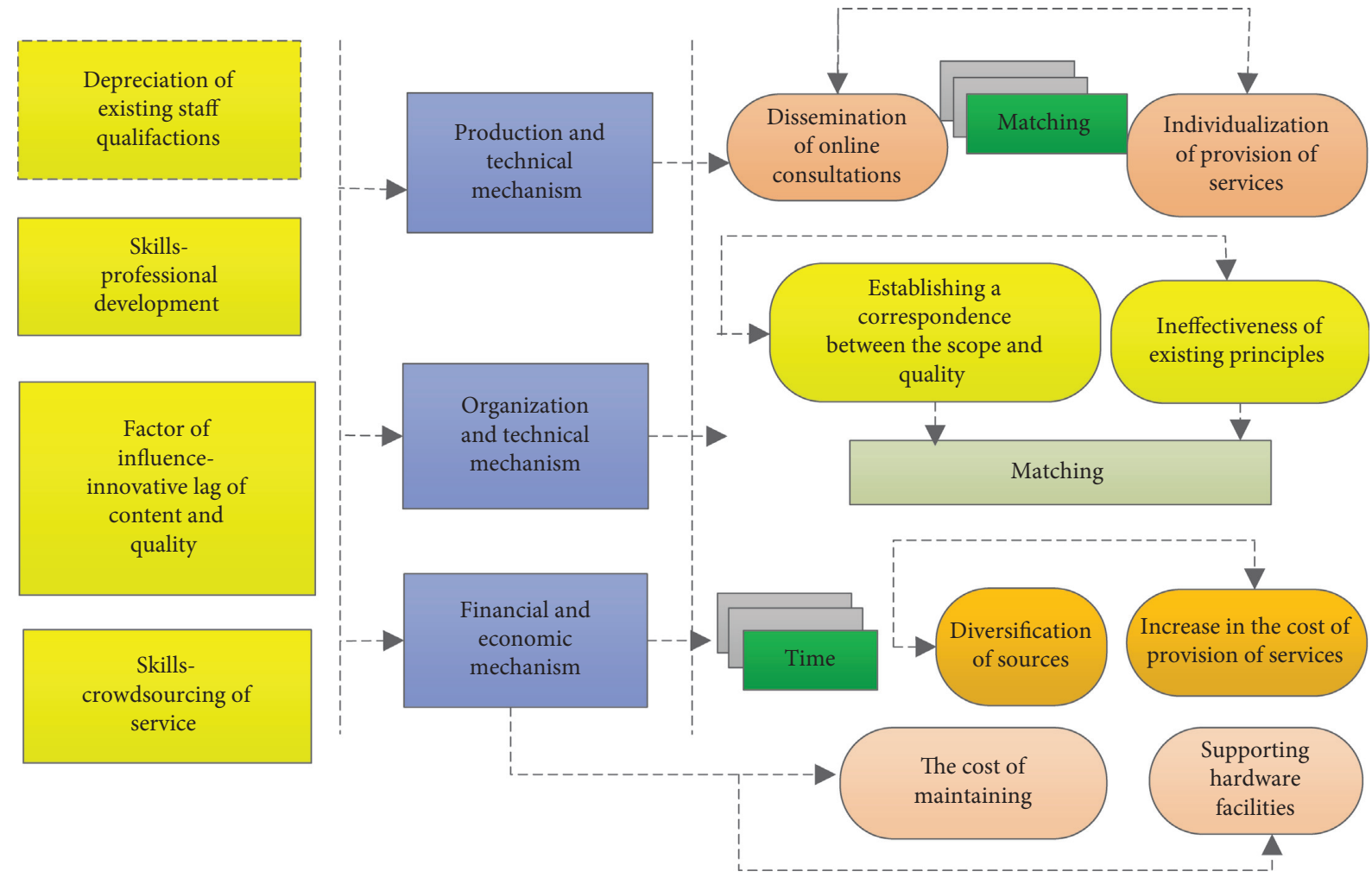

FIgURe 2: Conceptual model of person organization matching.

condition that the project duration and cost remain unchanged, so that the quality of each project is optimal, and customer satisfaction is maximized.

Suppose that the $j^{\text {th }}$ task of the $i$-th project requires the $k^{\text {th }}$ skill and needs to be completed by $l$ individuals. If $\left\{s_{1}, s_{2}, \ldots, s_{l}\right\}$ are assigned to do the task, the average skill level of labor resources in task $\chi_{k l}$ is

$$
f\left(x_{i j}\right)=\sum_{k=s_{1}}^{s_{l}} \chi_{k l} / l \text {. }
$$

The average skill level per unit time of project $y_{i}$ is

$$
f\left(y_{i}\right)=\frac{\sum_{j=1}^{n_{i}} f\left(x_{i j}\right) \varphi_{k l}}{\sum_{j=1}^{n_{i}} t_{i j}} .
$$

The labor resource skill level of all projects in the entire enterprise is

$$
f(Y)=\sum_{i=1}^{n} f\left(y_{i}\right) W_{i} .
$$

Among them, $\sum_{i=1}^{n} W_{i}=1, \sum_{j=1}^{n_{i}} \varphi_{i j}=1$.

The project quality optimization problem, that is, the objective function of the problem with the largest labor resource skill level allocated by all projects in the enterprise, is $\max f(Y)$, which means that the labor resource skill level of all projects per unit time is the strongest, and the project quality is the best. In order to ensure the optimal project quality, when allocating labor resources, tasks with larger project weights and task quality impact factors should be used to obtain labor resources with a higher skill level. The more important the tasks, the higher the skill level of the assigned labor resources.

\section{Empirical Analysis of Optimal Allocation of Enterprise Labor Resources}

5.1. Resource Optimization Allocation Algorithm. The first step is to initialize the program and define the matrix $M=\operatorname{initial}(m, t)$, which means that, before the task is assigned, $m$ individuals are free for $t$ days and can be used for the dispatch of these $m$ projects, where $t=\max \left(t_{i}\right)$, that is, the duration of the project with the longest duration in all projects; the definition matrix $X=\operatorname{initial}(m, n)$, which means that, before the task is assigned, all the tasks of the project have not been assigned to do it, where $n=\max \left(n_{i}\right)$, that is, the number of tasks of the project with the largest number of tasks in all projects.

The second step is to allocate human resources to the first task of each project and select personnel with high skill levels for deployment. When the $k$-th person was assigned to the first task of the $i$-th project in the previous $t_{i}$ day. Set $M(k, 1)$, $M(k, 2), \ldots, M\left(k, t_{i}\right)$ to 1 , and $X(k, 1)$ to $1 . M(k, t)=1$ means that the $k$-th person is in the working state on the $t$-th day. When the tasks of other projects in this time period require workers, they can only choose from other idle personnel. $X(k, 1)=1$ means that the first task of the first project is done by the $k^{\text {th }}$ person [21].

The third step is to assign human resources to the remaining tasks in turn. Before assigning labor resources to each task, first gather the personnel with $M\left(k, t_{i}\right)=0$ in the 
time period from $t_{1}$ to $t_{2}$ of the task to form an optional personnel set and select personnel with high skills. When the $k$-th person is assigned to the $j$-th task of the $i$-th project from day $t_{1}$ to $t_{2}$, set $M\left(k, t_{1}\right), M\left(k, t_{1}+1\right), \ldots, M\left(k, t_{2}\right)$ is 1 , and $X(k, n)$ is set to 1 . Then, when assigning human resources to subsequent tasks, you can only choose from the set of personnel with $M(k, t)=0$.

The fourth step is to output $F(Y)$ to obtain the labor resource allocation coefficient of all projects under the current resource allocation situation.

In Step 5, repeat steps 2, 3, and 4 until the maximum value of the objective function is obtained. And then, output the matrices $M$ and $X$ that maximize the objective function.

In last step, $M(m, t)$ and $X(m, n)$ are used to obtain the chart of each human resource assigned to each project task as shown in Figure 3.

\subsection{Multivariate Linear Regression Model Prediction. The} establishment of a multiple linear regression forecasting model is for the development trend of enterprise labor resource scale allocation. According to the current situation investigation and analysis, the correlation between the enterprise labor resource scale allocation and various technical and economic indicators will be related to the enterprise labor resource scale allocation from 2010 to 2017. Various economic and technical indicators with strong relevance are input into the SPSS software, and after screening, the multiple linear regression analysis model of the development trend of the labor resource scale allocation of enterprise can be output [22]; see formula (4):

$$
y=9579-483.3 x_{1}+3.28 \times 10^{-2} x_{2} .
$$

In the formula, $y$ is enterprise labor resource scale, $x_{1}$ is the year, and $x_{2}$ is the product sales profit.

After inspection, $R_{2}=0.923>R$ (when $P=0.05, n-2=9$, check the correlation coefficient critical table to get $R=0.602$ ); the Sig value of the equation is all less than 0.05 , which can pass the $t$-test: $F$ value is $18.532>F_{0.05}=4.46$. When a given significance level $a=0.05$, it indicates that the linear relationship of the model is significant, so the multiple linear regression equation can be used to predict the development trend of the scale of labor resources of the enterprise. The correlation coefficient test result is shown in Table 1.

\subsection{The Prediction Results of the Multiple Linear Regression} Model of the Scale of Enterprise Labor Resources. According to the strategic goal of enterprise, by 2018 and 2019, product sales profits will reach 600 million yuan and 1 billion yuan, respectively. According to the multiple linear regression analysis model of the development trend of enterprise labor resource scale allocation, it can be seen that the development trend of enterprise labor resource scale to 2018 and 2019 is shown in Table 2.

Using the above three different schemes to analyze the development trend of the scale of labor resource allocation of enterprise, each has its advantages and limitations. Based on the analysis results of the above three programs, the analysis results of the development trend of the labor resource scale allocation of enterprise are shown in Table 3.

The development trend is in line with the requirements of enterprise to control the scale, maintain dynamic balance, streamline the organization, and shift from the development of scale and quantity to the intensive development strategy of quality development, and it is also to improve the current allocation of labor resources of enterprise. The characteristics of the results of the analysis of the development trends of the scale allocation of labor resources of the three companies of the program are as follows. The analysis of the three patterns of development trends in enterprise labor resource scale allocation is down from the 2018 enterprise labor resource scale allocation. Labor resources in companies, on one hand, reflect the continuation of the trend of contraction and efficiency improvement that has recently formed due to the political factors of contraction and efficiency improvement. On the other hand, it reflects the impact of technological advances, the increase in labor productivity, and the reduction of labor intensity on the scale of a company's labor allocation.

\subsection{Examples of Optimization of Labor Resource Allocation.} A project-oriented enterprise has 3 projects that need to be implemented at the same time, the weight coefficient of each project $W=\{0.300,0.375,0.325\}$, each project has 4,5 , and 6 tasks, and the quality factor of each task and the labor required, the number of resources, required working hours, and skills required for implementation are shown in Table 4, Figures 4, and 5 respectively. In order to implement these 3 projects in the enterprise, there are 10 people available for labor resource allocation; and everyone has a different level of skill.

Among the three method analysis results of the development trend of labor resource scale allocation of enterprise, the gray system analysis result has the largest decline compared with the current situation of enterprise labor resource scale allocation in 2018.

On one hand, the production function model reflects the influence of technological progress factors and labor productivity on the scale of enterprises. On the other hand, compared to the situation in 2018, the result of the analysis of the production function model of the development trend of the company's labor allocation scale shows little decrease. The main reason for this trend is that the results of the production function model analysis of the development trend of the company's employee scale mainly reflect the relationship between labor, capital inflow, and production in the production function analysis model. These two reasons lead to a small decrease in the forecast results of the development trend of the scale of labor resource allocation of enterprise in 2018 and 2019. The skills required for each project task are shown in Figure 6.

As shown in Table 5 and Figure 7, the development trend of labor resource scale allocation of enterprise predicted by the multiple linear regression model is relatively stable. The 


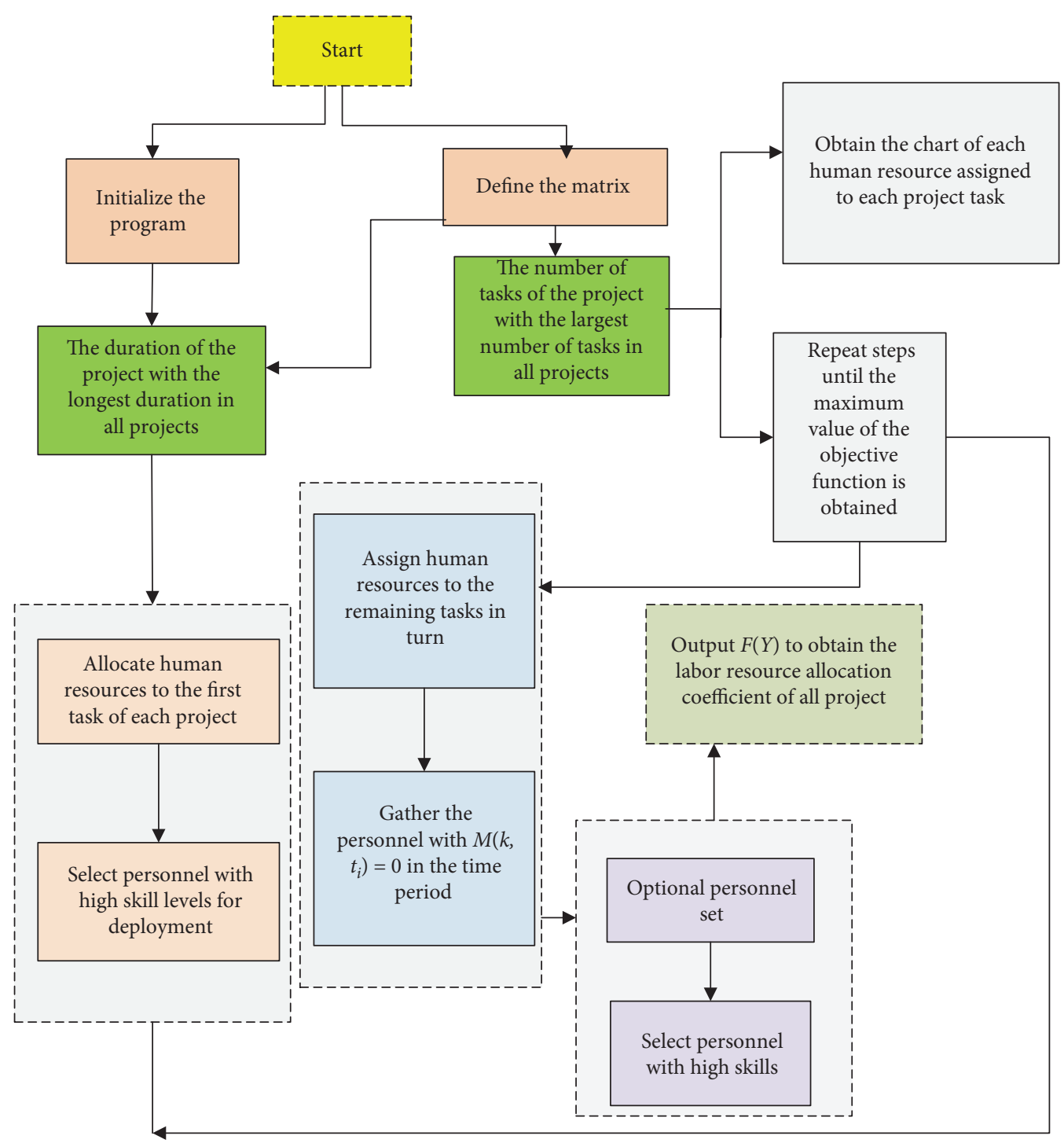

FIGURE 3: Resource optimization allocation algorithm.

TABLE 1: Correlation coefficient test result table.

Correlation coefficient

Coefficient of determination Adjust the coefficient of determination 0.923 0.872 0.656

TABLE 2: Statistics of the results of multiple linear regression analysis of the development trend of the scale of labor resource allocation in enterprise.

\begin{tabular}{lcc}
\hline Year & 2018 & 2019 \\
\hline Predication value & 76024 & 47619 \\
\hline
\end{tabular}

TABLE 3: Comparison table of three plans for the development trend of labor resource scale allocation in enterprise.

\begin{tabular}{lcc}
\hline Year/plan & 2018 & 2019 \\
\hline Gray system analysis & 48633 & 39473 \\
Production function analysis & 65403 & 60374 \\
Multiple linear regression analysis & 77463 & 56384 \\
\hline
\end{tabular}

multiple linear regression model predicts relatively stable results of the development trend of the scale of enterprise labor resource allocation. This is because the multiple linear regression predictive model combines the effects of two factors: attrition and efficiency. The trend of reducing staff and improving efficiency continues due to the influence of policy factors in recent years, not only reflecting that the scale of allocation of the workforce of companies is decreasing year by year, it reflects the impact of profits from product sales on the size of a company's workforce allocation and reflects the correlation between economic benefits and the size of employees as well. Due to the constraints of economic benefits, the model predicts the development of labor resource scale of company. 
TABLE 4: Task quality factors of each project.

\begin{tabular}{lcccccc}
\hline & Task 1 & Task 2 & Task 3 & Task 4 & Task 5 & Task 6 \\
\hline$W_{1}$ & 0.125 & 0.225 & 0.350 & 0.300 & 0 & 0 \\
$W_{2}$ & 0.300 & 0.200 & 0.250 & 0.200 & 0.050 & 0 \\
$W_{3}$ & 0.100 & 0.150 & 0.200 & 0.250 & 0.150 & 0.150 \\
\hline
\end{tabular}

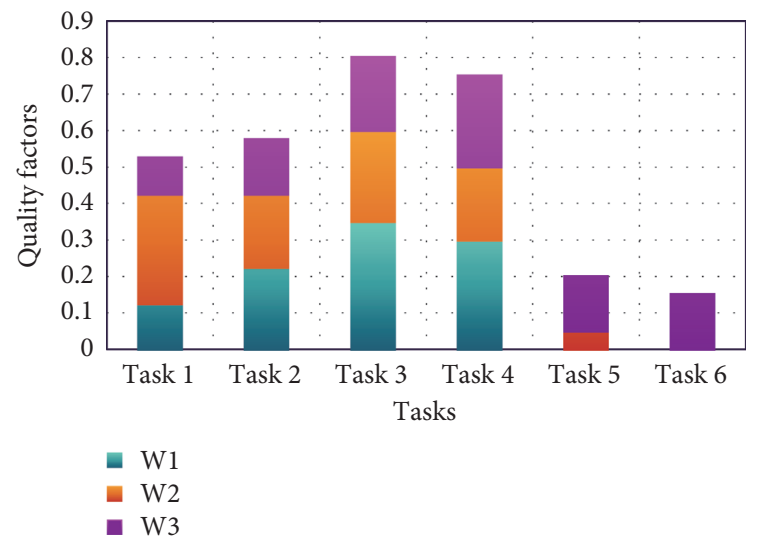

FIGURE 4: The quality factors of each project.

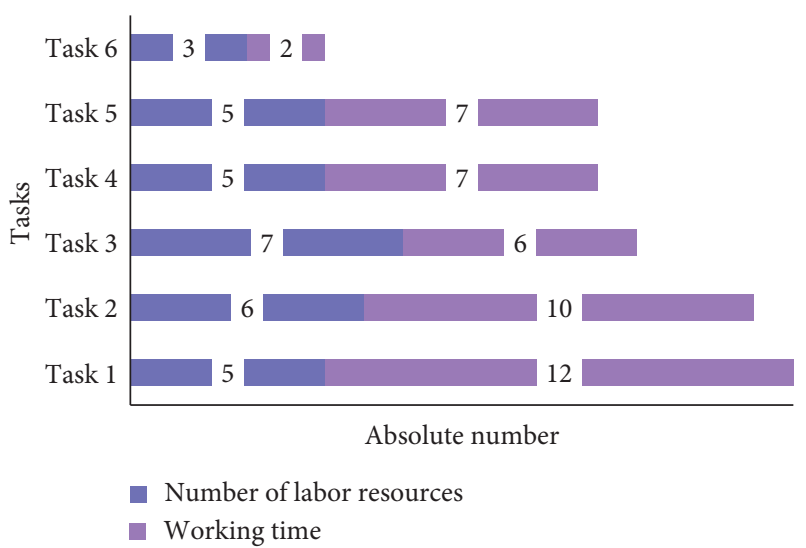

FIGURE 5: Number of labor resources and working time required for each project task.

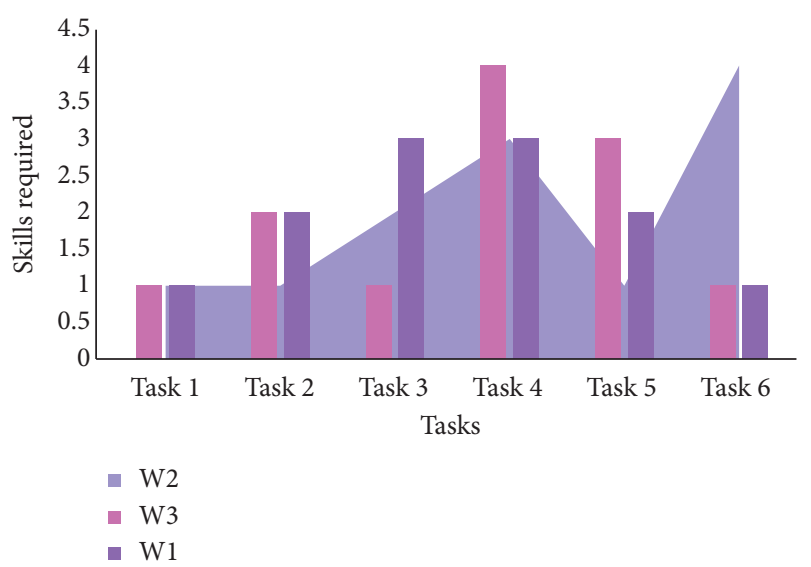

FIGURE 6: Skills required for each project task.
TABle 5: Coefficient of each skill level of each person.

\begin{tabular}{lcccc}
\hline Matching ID & Skill 1 & Skill 2 & Skill 3 & Skill 4 \\
\hline 1 & 0.65 & 1.24 & 0.26 & 0.62 \\
2 & 1.24 & 0.21 & 1.56 & 0.02 \\
3 & 1.14 & 1.21 & 1.45 & 1.04 \\
4 & 1.92 & 0.25 & 1.15 & 0.04 \\
5 & 1.07 & 0.26 & 0.88 & 0.01 \\
6 & 1.76 & 1.43 & 0.06 & 0.16 \\
7 & 1.09 & 0.76 & 0.91 & 0.18 \\
8 & 1.81 & 1.46 & 1.21 & 0.06 \\
9 & 0.17 & 0.97 & 0.89 & 0.26 \\
10 & 1.21 & 1.48 & 1.61 & 0.92 \\
\hline
\end{tabular}

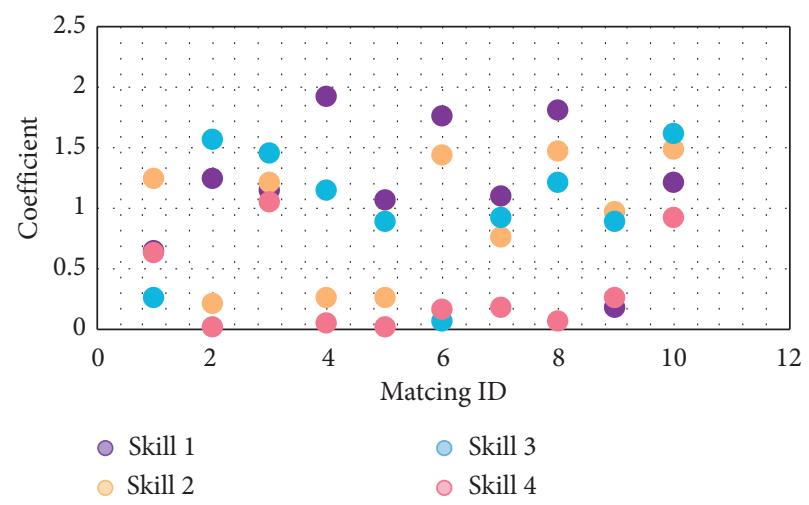

Figure 7: Coefficient of each skill level of each person.

From the above comprehensive analysis, the three kinds of forecasting plans for the development trend of the labor resource scale allocation of enterprises acan be seen, no matter what the theoretical basis or practical analysis are, as well as the development trend of the enterprise labor resource scale allocation in 2018 and 2019. The forecasts have good results.

\section{Conclusion}

Companies take project-based management as their organizational strategy, and project quality assurance plays a vital role in improving customer satisfaction and enhancing corporate image. The enterprise's human resource allocation needs to ensure that the quality of each project is optimized under the condition that the project duration and cost remain unchanged, and the customer satisfaction is maximized. From the perspective of optimizing project quality, this paper assigns different quality impact factors to each project and each task of a single project in a project-oriented enterprise and distinguishes the limited shared labor resources among multiple projects in the enterprise according to the skill level. Building an ideal work resource allocation model not only guarantees the quality of the project, but also brings long-term economic benefits to the project-oriented company, improves customer satisfaction, improves the corporate image, and facilitates the achievement of goals. There are many obstacles to artificially strengthening decision-making through empirical research on companies, 
and the bloated system, inefficiency, and low motivation for work are common problems for state-owned companies in our country. Measures have been taken in Asia to optimize the workforce of state-owned companies. The problem of project quality optimization is transformed into the problem of the greatest level of labor resource skill level for all project allocation of the enterprise, and the optimization of project quality is realized by realizing the optimal allocation of labor resources. The method of multiplan combination forecasting and evaluating the optimal allocation of enterprises shows that the ultimate goal of labor resource planning is to realize the balance of supply and demand of labor resources in quantity and quality through the forecast of supply and demand of labor resources of enterprises.

\section{Data Availability}

The data used to support the findings of this study are available from the corresponding author upon request.

\section{Conflicts of Interest}

The authors declare that they have no known competing financial interests or personal relationships that could have appeared to influence the work reported in this paper.

\section{Acknowledgments}

This research has partly benefited from financial support from National Social Science Foundation of China (19BGL270), Returned Talent Foundation of Hebei Province (C20200323), and Social Science Foundation of Hebei Province (HB19GL042).

\section{References}

[1] A. Olga, Z. Olga, and O. Julia, "The optimization of business processes at the enterprises of agro-industrial complex," International Multidisciplinary Scientific GeoConference: SGEM, vol. 19, no. 5, pp. 863-868, 2019.

[2] V. A. Varma, G. V. Reklaitis, and G. E. Blau, "Enterprise-wide modeling \& optimization-an overview of emerging research challenges and opportunities," Computers \& Chemical Engineering, vol. 31, no. 6, pp. 692-711, 2007.

[3] K. B. Aviso, A. P. Mayol, M. A. B. Promentilla et al., "Allocating human resources in organizations operating under crisis conditions: a fuzzy input-output optimization modeling framework," Resources, Conservation and Recycling, vol. 128, no. 8, pp. 250-258, 2018.

[4] W. Gu, T. L. Saaty, and L. Wei, "Evaluating and optimizing technological innovation efficiency of industrial enterprises based on both data and judgments," International Journal of Information Technology \& Decision Making, vol. 17, no. 1, pp. 9-43, 2018.

[5] W. Rankothge, F. Le, A. Russo, and J. Lobo, "Optimizing resource allocation for virtualized network functions in a cloud center using genetic algorithms," IEEE Transactions on Network and Service Management, vol. 14, no. 2, pp. 343-356, 2017.

[6] X. Wang and Z. Yang, "Application of fuzzy optimization model based on entropy weight method in atmospheric quality evaluation: a case study of Zhejiang province, China," Sustainability, vol. 11, no. 7, pp. 2143-2144, 2019.

[7] W. Zhang, S. Zhang, S. Guo, Y. Yang, and Y. Chen, "Concurrent optimal allocation of distributed manufacturing resources using extended teaching-learning-based optimization," International Journal of Production Research, vol. 55, no. 3, pp. 718-735, 2017.

[8] W. Zhao, Q. Zeng, G. Zheng, and L. Yang, "The resource allocation model for multi-process instances based on particle swarm optimization," Information Systems Frontiers, vol. 19, no. 5, pp. 1057-1066, 2017.

[9] E. Aghamohammadzadeh, M. Malek, and O. F. Valilai, "A novel model for optimisation of logistics and manufacturing operation service composition in cloud manufacturing system focusing on cloud-entropy," International Journal of Production Research, vol. 58, no. 7, pp. 1987-2015, 2020.

[10] E. Panfilova, N. Dzenzeliuk, O. Domnina et al., "The impact of cost allocation on key decisions of supply chain participants," International Journal of Supply Chain Management, vol. 9, no. 1, pp. 552-558, 2020.

[11] J. Leng, D. Yan, Q. Liu et al., "ManuChain: combining permissioned blockchain with a holistic optimization model as bi-level intelligence for smart manufacturing," IEEE Transactions on Systems, Man, and Cybernetics: Systems, vol. 50, no. 1, pp. 182-192, 2019.

[12] H. Wu, G. K.-H. Pang, K. L. Choy, and H. Y. Lam, "Dynamic resource allocation for parking lot electric vehicle recharging using heuristic fuzzy particle swarm optimization algorithm," Applied Soft Computing, vol. 71, pp. 538-552, 2018.

[13] W. Xu and Y. Yin, "Functional objectives decision-making of discrete manufacturing system based on integrated ant colony optimization and particle swarm optimization approach," Advances in Production Engineering \& Management, vol. 13, no. 4, pp. 389-404, 2018.

[14] P. Tomar, R. Mishra, and K. Sheoran, "Prediction of quality using ANN based on Teaching-Learning Optimization in component-based software systems," Software: Practice and Experience, vol. 48, no. 4, pp. 896-910, 2018.

[15] A. Naseri and N. J. Navimipour, "A new agent-based method for QoS-aware cloud service composition using particle swarm optimization algorithm," Journal of Ambient Intelligence and Humanized Computing, vol. 10, no. 5, pp. 1851-1864, 2019.

[16] A. Evangelinou, M. Ciavotta, D. Ardagna, A. Kopaneli, G. Kousiouris, and T. Varvarigou, "Enterprise applications cloud rightsizing through a joint benchmarking and optimization approach," Future Generation Computer Systems, vol. 78, no. 8, pp. 102-114, 2018.

[17] L. Guo and J. Qiu, "Optimization technology in cloud manufacturing," The International Journal of Advanced Manufacturing Technology, vol. 97, no. 1-4, pp. 1181-1193, 2018.

[18] F. Liu, T. Lv, M. Sajid, and X. Li, "Optimization for China's coal flow based on matching supply and demand sides," Resources, Conservation and Recycling, vol. 129, no. 9, pp. 345-354, 2018.

[19] H. Yu and W. D. Solvang, "A carbon-constrained stochastic optimization model with augmented multi-criteria scenariobased risk-averse solution for reverse logistics network design under uncertainty," Journal of Cleaner Production, vol. 164, pp. 1248-1267, 2017.

[20] J. Wang, A. Liu, T. Yan, and Z. Zeng, "A resource allocation model based on double-sided combinational auctions for transparent computing," Peer-to-Peer Networking and Applications, vol. 11, no. 4, pp. 679-696, 2018. 
[21] J. Jin and W. Mi, "An AIMMS-based decision-making model for optimizing the intelligent stowage of export containers in a single bay," Discrete \& Continuous Dynamical Systems, vol. 12, no. 5, pp. 1101-1103, 2019.

[22] G. Qin, F. Tao, and L. Li, “A vehicle routing optimization problem for cold chain logistics considering customer satisfaction and carbon emissions," International Journal of Environmental Research and Public Health, vol. 16, no. 4, pp. 576-577, 2019. 